\title{
Interculturalidad en el Desarrollo de la Identidad Nacional en estudiantes del Colegio Emblemático № 56001 “Mateo Pumacahua”, Sicuani-Cusco
}

\author{
Interculturality in the Development of National Identity in Students of the Emblematic College No. 56001 \\ "Mateo Pumacahua", Sicuani-Cusco
}

Interculturalidade no Desenvolvimento da Identidade Nacional em Alunos do Colégio Emblematico № 56001

"Mateo Pumacahua", Sicuani-Cusco

\author{
Clotilde Araoz Enriquez \\ clotildearaozenriquez@gmail.com \\ https://orcid.org/0000-0002-4555-9719 \\ Norka Inés Obregón Alzamora
nobregon@unfv.edu.pe
https://orcid.org/0000-0002-7147-3548
}

Luis Fernando Blanco Ayala

lblanco@unmsmm.edu.pe

https://orcid.org/0000-0003-3211-1269

Universidad Nacional Mayor de San Marcos - UPG, Lima-Perú

Artículo recibido en junio 2021, revisado en julio 2021, arbitrado en agosto 2021 y publicado en septiembre 2021

\section{RESUMEN}

El presente artículo contribuye al análisis e interpretación de la interculturalidad y la identidad nacional en estudiantes para reflexionar sobre las políticas educativas. El objetivo principal es determinar la influencia entre las variables. La investigación es cuantitativa. La muestra se determinó por muestreo aleatorio simple, a quienes se aplicó la fórmula para determinar el tamaño de la muestra 51 profesores y 217 estudiantes. Se diseñaron dos instrumentos de recopilación de datos y para su aplicación se sometieron a pruebas de confiabilidad estadística para ello se utilizó el procedimiento estadístico: coeficiente de Alfa de Cronbach. Los resultados demuestran que los aspectos interculturales aplicados por los maestros durante la enseñanza, son buenos y promueven el desarrollo de la Identidad Nacional de los estudiantes. Concluimos que la Interculturalidad utilizada por los maestros influye significativamente en el desarrollo de la Identidad Nacional en estudiantes del Colegio Emblemático $\mathrm{n}^{\circ}$ 56001 "Mateo Pumacahua", Sicuani-Cusco.

Palabras clave: Diversidad cultural; Identidad cultural; Integración cultural; Interculturalidad

\section{ABSTRACT}

This article contributes to the analysis and interpretation of interculturality and national identity in students, to reflect on educational policies. The main objective is to determine the influence between the variables. The research is quantitative. The sample was determined by simple random sampling, to whom the formula was applied to determine the sample size of 51 teachers and 217 students. Two data collection instruments were designed and for their application, they were subjected to statistical reliability tests. For this, the statistical procedure was used: Cronbach's alpha coefficient. The results show that the intercultural aspects applied by teachers during teaching are good and promote the development of the students' National Identity. We conclude that the Interculturality used by the teachers significantly influences the development of the National Identity in students of the Emblematic College No. 56001 "Mateo Pumacahua", Sicuani-Cusco.

Key words: Cultural diversity; Cultural identity; Cultural integration; Interculturality

\section{RESUMO}

Este artigo contribui para a análise e interpretação da interculturalidade $e$ identidade nacional em alunos, para refletir sobre as políticas educacionais. $\mathrm{O}$ objetivo principal é determinar a influência entre as variáveis. A pesquisa é quantitativa. A amostra foi determinada por amostragem aleatória simples, à qual foi aplicada a fórmula para determinar o tamanho da amostra de 51 professores e 217 alunos. Foram elaborados dois instrumentos de coleta de dados e, para sua aplicação, foram submetidos a testes de confiabilidade estatística, para isso foi utilizado o procedimento estatístico: coeficiente alfa de Cronbach. Os resultados mostram que os aspectos interculturais aplicados pelos professores durante a docência são bons e promovem o desenvolvimento da Identidade Nacional dos alunos. Concluímos que a Interculturalidade utilizada pelos professores influencia significativamente no desenvolvimento da Identidade Nacional nos alunos do Colégio Emblematico nº 56001 "Mateo Pumacahua", Sicuani - Cusco

Palavras-chave: Diversidade cultural; Identidade cultural; Integração cultural; Interculturalidade 


\section{INTRODUCCIÓN}

La identidad es la conciencia que una persona tiene respecto a ella misma y que la convierte en alguien distinta a los demás. Aunque muchos de los rasgos que forman la identidad son hereditarios o innatos, el entorno ejerce influencia en la conformación de la especificidad de cada sujeto. La identidad constituye también un sistema de símbolos y de valores que permite afrontar diferentes situaciones cotidianas. Es el conjunto de rasgos propios de un individuo o de una comunidad. En esta zona del Perú (Cusco) El idioma materno "el quechua", no solo debe ser útil para comunicarse desde que se nace, sino también sirve para adquirir nuevos conocimientos. El Ministerio de Educación ha anunciado un mayor soporte pedagógico en 4000 colegios de educación intercultural bilingüe (EIB), en los que se hablan algunos de los 47 dialectos existentes en Perú. (Diario el Peruano, 2017).

El Plan Nacional de Educación Intercultural Bilingüe es un documento de gestión que implementa la Política Nacional de Educación Intercultural Bilingüe. En la que se considera todos los componentes y ejes de la política planteando las metas, resultados, estrategias e indicadores de ejecución, hasta el 2021. En la investigación se incluyó tres indicadores que buscarán asegurar el cumplimiento del propósito del plan nacional de educación intercultural bilingüe: La entrega oportuna de materiales educativos en lenguas originales; maestros que reciban acompañamiento pedagógico, y tención en el servicio EIB.

Este apoyo es vital porque en el país existen 24,964 instituciones educativas bilingües, ubicadas en su mayoría en la Amazonía, al igual que en Cusco, Apurímac y Ayacucho de Perú. Atienden a unos 100. 200. 000 niños, de los cuales el mayor porcentaje está en primaria. (Diario el Peruano, 2017). Bajo esta premisa el objetivo es fortalecer el nivel de desarrollo de las competencias interculturales, como repuesta a la inquietud para promover la identidad nacional en estudiantes del Colegio Emblemático no 56001 "Mateo Pumacahua", Sicuani-Cusco en Perú, ahora bien, la importancia desde el punto de vista social, plantea la formación de un ciudadano intercultural con convicciones democráticas y colaborativas con una nueva moral que participe activamente en la edificación de una sociedad equitativa, incluyente y desarrolladora.

\section{Interculturalidad}

La interculturalidad se refiere a la relación, diálogo e interacción dinámica de las personas en su cultura. Significa promover el espacio de diálogo, discusión, debate y consenso sobre estos temas entre profesores y estudiantes con el fin de obtener el mejor enfoque. Debe ir desde la realidad de la alienación a la diversidad cultural inherente a nuestra sociedad, o simplemente el encuentro entre culturas; implica saber reconocer la diversidad positiva de las culturas e interactuar con ellas para lograrlo en nuestro entorno real y nuestras necesidades de aprender. Aguado (2005) afirma que la interculturalidad no es solo una verificación de la convivencia cultural, sino que también expresa el establecimiento de un cierto tipo de relación entre culturas. Es decir, cuando se trata de interculturalidad, primero se asume que los profesores deben tener una hipótesis, organizar y orientar las actividades docentes El propósito es gestionar la existencia de contenidos culturales mixtos en un mismo espacio y tiempo, pertenecientes a diferentes culturas.

Ciertamente, se trata de reducir algunos problemas o dificultades en el ámbito educacional que generan situaciones de desigualdad y discriminación social. Entonces, para una 
intervención eficaz áulica e institucional, es crucial que el docente conozca con claridad la realidad y contexto educativo y elabore una planificación, basado en un modelo pedagógico respecto a la cual fundamentara su intervención y desempeño. Walsh (2009) plantea cinco afirmaciones respecto a la interculturalidad: en condiciones de respeto, legitimidad mutua, simetría e igualdad, el proceso dinámico y permanente de relaciones, comunicación y aprendizaje entre culturas; establecer intercambios entre personas, saberes, saberes y prácticas de diferentes culturas, buscando cultivar un nuevo sentido de convivencia en sus diferencias; un espacio de negociación y traducción La desigualdad social, económica y política, así como la relación y los conflictos de poder en la sociedad no se ocultarán, sino que serán reconocidos y enfrentados; desafiar las tareas sociales y políticas de toda la sociedad, partiendo de prácticas y acciones sociales concretas y conscientes, $y$ tratando de crear un modelo de responsabilidad y solidaridad, y un objetivo a alcanzar.

La interculturalidad promueve la integración social, defiende las relaciones humanas basadas en la tolerancia de las diferencias, en la empatía de aquello que nos hace distintos, es decir, apertura perspectivas de intercambio y desarrollo de todo tipo; es una prioridad como enfoque social y educativo. Somos conscientes que las sociedades de nuestro tiempo no pueden avanzar libre de la integración. Por su parte INDEPA (2010), plantea que la interculturalidad promueve "relaciones de confianza, reconocimiento mutuo, comunicación efectiva, comprensión del otro desde su propia cultura, dialogo y debate, intercambio de saberes y experiencias, resolución pacífica de conflictos, consensos desde las diferencias, cooperación y convivencia" (p.47).
Promover la Interculturalidad en los estudiantes del Colegio Emblemático no 56001 "Mateo Pumacahua", Sicuani-Cusco, implica desarrollar el trabajo colaborativo, la cooperación, el trabajo en redes mediante el uso adecuado, intencional y sistemático de herramientas integradoras como la comunicación asertiva y empática, constituyen en estos tiempos, pilares de desarrollo humano. Todo ello, con el trabajo planificado y la diversificación curricular incluyendo diferentes actividades con la participación conjunta de docentes y estudiantes.

\section{Perfil del profesor intercultural}

El profesor es la figura por excelencia del instrumento pedagógico, es pieza clave para la educación de calidad, se resalta la importancia del ser y quehacer del profesorado. Aguado (2005) afirma que, el rol del profesor es desarrollar una educación intercultural. Por ello, deben recibir formación inicial, continua y permanente de forma que estén capacitados con cada nuevo grupo de estudiantes y que les permita reconocer necesidades específicas de sus estudiantes y valorar a los aportes de determinados alumnos y de sus familias en las instituciones educativas.

Es necesario que todos los implicados en el rol educativo actúen como miembros activos para el desarrollo del aprendizaje de los estudiantes; de esta forma, mediante diversas actividades se debe buscar estimular el aprendizaje cooperativo, las interacciones entre estudiantes y profesores, la reflexión y autoaprendizaje. Considerando las diferencias de los estudiantes en cuanto estilos de aprendizaje, motivacionales y comunicativos. Fernández (2008) señaló que la imagen del docente intermediario en el proceso de enseñanza, basada en las diferencias culturales, debe comprender situaciones de aprendizaje con diversos elementos 
culturales, y orientar a los estudiantes para que desarrollen sus habilidades y habilidades reflexivas en torno a la realidad cultural, para orientarlos. Dar pasos positivos de forma activa, posicionar y reconocer la diversidad cultural.

A pesar de las diferencias culturales, sociales y de género, la educación intercultural es la posibilidad de desarrollar plenamente las capacidades individuales. Salazar y Schimtz (2015) estimaron que los docentes deben alentar a los estudiantes a comprender su cultura y las diversas culturas con las que interactúan; los docentes deben hacer un pre-diagnóstico, identificar y recolectar las costumbres de los estudiantes, información de contacto y métodos de aprendizaje. De esta manera, desarrollar estrategias que contribuyan a la armonía social y eliminen la derogación cultural. Es aprender de encuentros e interacciones con personas pertenecientes a diferentes culturas, porque él o ella es miembro de la comunidad; se debe enseñar a compartir el conocimiento de todos para enriquecer a otro con conocimiento ancestrales propios de Perú, por lo que primero en necesario entender la identidad.

La imagen de un profesor intercultural no solo enfatiza su papel en el aula o la necesidad de una formación continua y permanente, sino que también enfatiza las actitudes y habilidades que debe poseer. Por lo tanto, es imperativo tener múltiples actitudes y habilidades específicas. Promover la educación intercultural de los estudiantes requiere esencialmente una mente abierta y estándares flexibles, reflexionando constantemente sobre lo que observan y tomando decisiones basadas en sus propios juicios críticos. Debe desarrollar actividades y estrategias de enseñanza para la tolerancia cultural, por lo tanto, debe reconocer y reflexionar sobre propias culturas locales, regionales y nacionales, para luego enriquecer las diversas culturas con las que se interactúan. Entonces, el profesor debe recurrir a estrategias didácticas para poder promover el desarrollo de la identidad cultural en los estudiantes para brindar un ambiente escolar seguro; promover espacios y oportunidades para generar el propio conocimiento; inducir al estudiante a comprender su adquisición de identidad cultural; explorar estrategias para desarrollar en el estudiante un agente de cambio, la eficacia del docente depende del evitar la influencia de estereotipos de determinados grupos culturales.

Con ello se afirma que el estudiante es el agente principal en su propio aprendizaje, a partir de sus intereses el docente lo guiará a través de espacios $\mathrm{y}$ oportunidades, inclusive interviniendo cuando observe alguna conducta no deseada para lograr los objetivos de la educación intercultural. Surge la idea de la relación entre culturas, intercambio de saberes, habilidades y formas de ser. Los estudios interculturales son aplicados en el ámbito de la educación en regiones y países multiculturales. Perú es un escenario que ofrece una diversidad cultural bastante marcada por sus regiones. La nación está dividida básicamente en tres zonas: costa, sierra y selva. En algunos casos existe fusión entre una y otra localidad geográfica, esto hace que concurran algunas coincidencias en sus costumbres, pero, con marcadas diferencias en sus idiosincrasias y formas de vivir según sus culturas propias como ritos, economías, costumbres y lenguas. Arribar la interculturalidad implica las siguientes actividades:

\section{Prácticas culturales}

El sistema educativo implica que se asume la diversidad cultural desde una perspectiva de respeto y equidad social, una perspectiva que todos los sectores de la sociedad tienen que asumir hacia los otros. Un aspecto que permite identificar las buenas prácticas culturales, es el hecho de someter 
a opinión y juicio de los estudiantes del Colegio Emblemático no 56001 "Mateo Pumacahua", Sicuani-Cusco en Perú, sobre la interculturalidad.

\section{Uso del idioma quechua}

En estos últimos tiempos en contextos de interculturalidad para fortalecer la enseñanza y el aprendizaje es un aspecto crucial. Frecuentemente el uso del idioma materno quechua es de carácter doméstico, es decir se acostumbra el uso solamente en el seno familiar, basada en las costumbres, tradiciones, creencias y valores propios de las distintas familias y dependiendo del lugar donde provienen, frecuentemente de zonas de periferia. En tiempos anteriores el idioma quechua original materno de la zona del Cusco, era muy usual inclusive como el medio más útil en la educación en el entorno familiar y en las escuelas de aquel entonces, hoy por hoy estas costumbres se están perdiendo. Somos conscientes que las lenguas maternas son recursos educativos fundamentales, ya que destacan para enseñar a valorar la cultura de los pueblos que se están perdiendo.

\section{Procesos de convivencia, cooperación y autoestima.}

Estos procesos son fundamentales en el Colegio Emblemático $\mathrm{n}^{\circ} 56001$ "Mateo Pumacahua", Sicuani Cusco-Perú, permite convivir en armonía supone el respeto a las ideas y sentimientos de los demás, promueve la tolerancia frente a la diversidad intercultural, fortalece la solidaridad, reciprocidad y cooperación mutua entre estudiantes, docentes, padres de familia, autoridades y otros de la comunidad educativa todos con un objetivo común. En tal sentido, todas las instancias (padres, estudiantes, docentes y autoridades), confluyen de manera colaborativa en las diferentes actividades programadas a nivel institucional. Nuestros padres de familia en los últimos tiempos, muestran su vinculación directa en la formación de sus hijos, ellos participan en todos los proyectos apoyando a sus hijos, fortaleciendo la convivencia escolar.

\section{Desarrollo de la identidad nacional}

Al intentar definir la identidad nacional, que teóricamente se inserta en el concepto de identidad social. No se trata de un hecho simple unilateral, se trata de diferentes categorías las que puede resultar una abstracción un tanto compleja. Aun cuando un pueblo, región o nación es altamente potencial en diversidad de costumbres, etnias y culturas, entre otros factores. En otras palabras, tiene un sentido de dominio sobre un grupo de personas relacionado con ciertas características culturales, institucionales e históricas, además de aspectos de evaluación y emocionales. Pastor (2006) afirmó que la identidad nacional significa ser un catalizador de la afiliación, que a su vez hace que los individuos de la sociedad se sientan leales no solo a su familia, a su gente o su ciudad, sino que también es influenciado por otras comunidades culturales del país, y mantiene diversos grados de identidad con ellas.

Perú no es ajeno a esta complejidad, algunos expertos creen que el concepto de nación es relativo o aún en construcción. En este sentido, Romero (2015) afirmó que definir la identidad nacional es una tarea compleja porque se expresa como una abstracción geopolítica, más que como un compromiso unificado de pertenencia al país. Este antecedente ha enriquecido a los peruanos como una impresionante riqueza de diversidad y orgullo en un contexto transcultural. El tema de la identidad nacional de Perú puede convertirse en una deuda histórica. Cueto, Espinosa y Robles (2017); manifestaron que identificaron una situación problemática que se remonta a la fundación de la República, y es difícil consolidar 
un sentimiento nacional positivo. El rechazo de los indígenas peruanos en la formación del país muestra que el rechazo a la diversidad étnica es un rasgo común de los antiguos territorios coloniales. La concienciación nacional se considera una tarea abrumadora, especialmente en el contexto del racismo y la marginación.

En la actualidad, la exclusión de la clase media limeña frente a los pueblos andinos e indígenas es un claro representante de la sociedad peruana, es el resultado de diferentes y continuos conflictos internos en diversos ámbitos de la sociedad peruana, y muchas veces es promovida por los representantes de la actualidad política. Debido a intereses prejuiciosos individualizados, su único resultado es el deterioro de identidad nacional. Existen diferentes estudios sobre "identidad nacional", Beramendi (2020) afirmó que tiene un impacto negativo en la construcción del autoconcepto colectivo y la autoestima del sujeto. Espinosa et al. (2016) caracterizan la identidad nacional como un aspecto contradictorio o ambiguo. Los peruanos no son tan buenos como quieren, lo que se puede expresar como una identidad negativa.

Para ello, es necesario desarrollar un conjunto de estándares internos en torno a los deseos de cada miembro de la comunidad educativa, teniendo en cuenta los diferentes antecedentes interculturales, las autoevaluaciones y el pensamiento crítico de las personas (individuos y grupos). Valorar la identidad étnica y recoger el patrimonio cultural de la región, e integrar: las normas, valores, lengua y costumbres quechuas, unificarlas o distinguirlas. Son una actitud positiva que hay que fortalecer constantemente, valorante asimismo primero y luego a los demás. En este marco, con el aporte del sector educativo y la participación activa y voluntaria, se han formulado una serie de estrategias para evitar conflictos de identidad cultural entre los estudiantes, cuidarse a sí mismos, tener autonomía, igualdad y equidad. Por lo tanto, la identidad nacional es un proceso que involucra múltiples interacciones $y$, como parte de la sociedad, tiene diferentes consecuencias a nivel individual y grupal.

Considerando la experiencia exitosa y positiva en el tema de la "identidad nacional", como un proceso de múltiples interacciones, entre los estudiantes de la escuela simbólica Sicuani-Cusco No. 56001 "Mateo Pumacahua” Perú, se propusieron las siguientes actividades:

\section{Identificación con los símbolos patrios}

Son los componentes que identifican a Perú, que representan situaciones trascendentales de la riqueza histórica, logros, y recursos naturales. Bueno, Molina y Porras (2009), afirma que: "los símbolos patrios representan la autonomía y el carácter oficial de la nacionalidad. Son íconos que identifican al país y lo distinguen de cualquier otra nación del mundo". Son fundamentales los símbolos patrios porque permite que la ciudadanía en general se identifique con ellos. Los ciudadanos consiguen unirse bajo el contexto de la misma simbología que representa el país. En el Colegio Emblemático n ${ }^{\circ}$ 56001 "Mateo Pumacahua", Sicuani, Cusco-Perú, vienen desarrollándose una serie de actividades interculturales para fortalecer la identificación con los símbolos patrios y promover la identidad nacional, que en los últimos tiempos viene siendo muy resquebrajada.

\section{Identificación con los monumentos históricos}

Son artefactos o construcciones de carácter cultural dentro de un pueblo y región que marcan un contexto histórico del país, permite la atribución de un valor en particular de acuerdo al tiempo y desde una perspectiva histórica. En el Cusco, existen muchos monumentos históricos, que permiten 
revalorar el legado de la historia de Perú antiguo de esta parte de la región, entre ellos mencionamos algunos que resaltan: la Piedra de los doce ángulos; Casa del gobierno: El muro inca: Machu Picchu: Valle Sagrado de los Incas: Tempo de Qorikancha: Amaru Cancha: Sacsayhuamán: Tambomachay. Son monumentos más representativos del Cusco-Perú, es decir, existen mucho más y todos representan un altísimo valor artístico, histórico, social. Por ello, a través de las diferentes actividades que se desarrollan en el Colegio Emblemático no 56001 "Mateo Pumacahua", Sicuani, Cusco-Perú, los estudiantes participan activamente, se promueve e incentiva la identificación de los monumentos históricos como parte de la identidad nacional. Actualmente, estos monumentos históricos son obras públicas, declaradas monumentos históricos por ley, y están protegidas por el Estado Peruano, representan el patrimonio cultural de nuestra nación con una enorme importancia sociocultural.

\section{Identificación con los personajes}

Es fundamental que los estudiantes presenten identidad a ciertos personajes de la historia del Perú y más aún si son parte de la historia de vuestra zona. Los hechos del pasado se interpretan frecuentemente en el contexto de una compleja red de relaciones causales y motivacionales, por ello como plan de enseñanza y aprendizaje se elaboran textos rescatando el contexto real de la zona. La experiencia permite afirmar que los estudiantes tienden otorgar mayor importancia a personajes más cercanas en el tiempo respecto a las más remotas, por ello a través de las experiencias exitosas, se socializa en los estudiantes la importancia de revalorar a los personajes de la historia de Perú a pesar de la distancia en el tiempo, debido a que son forjadores de la patria.

\section{Funciones de la identidad nacional}

Al respecto, Pérez (2012), afirma las siguientes funciones, desde el punto de vista político, es la base del Estado y sus instituciones y legitima los derechos y obligaciones legales comunes estipuladas por las instituciones. Estos derechos y obligaciones definen las características y valores especiales de la nación y reflejan las tradiciones, costumbres y costumbres de la gente; en cuanto a la sociedad, se le ha dado una mayor tolerancia, legitimando objetivos políticos y medidas administrativas que regulan la vida de los ciudadanos, desde la perspectiva de la Hermandad, considere las alianzas pasadas, presentes y futuras que existen entre familias, comunidades étnicas y naciones. Estas acciones están diseñadas para recordar a las personas sus lazos culturales y políticos y reafirmar la identidad y la unidad nacional.

\section{MÉTODO}

La estrategia de análisis metodológico diseñada para el estudio fue de tipo descriptivoexplicativo, enfoque cuantitativo, dado que el interés de los investigadores el cual estuvo centrado exclusivamente en establecer el nivel de influencia que existe entre las dos variables (Interculturalidad vs. Identidad nacional) en docentes y estudiantes sujetos de la muestra de estudio del Colegio Emblemático no 56001 "Mateo Pumacahua", Sicuani, Cusco-Perú. (Tabla 1). 
Tabla 1. Interculturalidad vs. Identidad nacional.

\begin{tabular}{|c|c|c|c|c|c|c|c|c|c|c|}
\hline \multirow[b]{2}{*}{ Total alumnos } & \multicolumn{2}{|c|}{$1^{\circ}$ grado } & \multicolumn{2}{|c|}{$2^{\circ}$ grado } & \multicolumn{2}{|c|}{$3^{\circ}$ grado } & \multicolumn{2}{|c|}{$4^{\circ}$ grado } & \multicolumn{2}{|c|}{$5^{\circ}$ grado } \\
\hline & $\mathbf{H}$ & $\mathbf{M}$ & H & $\mathbf{M}$ & $\mathbf{H}$ & $\mathbf{M}$ & $\mathbf{H}$ & $\mathbf{M}$ & H & $\mathbf{M}$ \\
\hline & 212 & 211 & 234 & 220 & 216 & 185 & 192 & 194 & 172 & 164 \\
\hline 2000 & \multicolumn{2}{|c|}{423} & \multicolumn{2}{|c|}{454} & \multicolumn{2}{|c|}{401} & \multicolumn{2}{|c|}{386} & \multicolumn{2}{|c|}{336} \\
\hline $\mathbf{n}=$ & \multicolumn{2}{|c|}{100} & \multicolumn{2}{|c|}{100} & \multicolumn{2}{|c|}{100} & \multicolumn{2}{|c|}{100} & \multicolumn{2}{|c|}{100} \\
\hline
\end{tabular}

$\mathrm{n}=$ se determinó 100 estudiantes de cada grado sumando a un total de 500 estudiantes.

La muestra con la cual se trabajó es: 58 docentes y 500 estudiantes. Los instrumentos de investigación fueron dos encuestas la técnica de investigación es la observación.

\section{RESULTADOS Y DISCUSIÓN}

Se plantean las siguientes hipótesis de trabajo, en base a la hipótesis general:
Hi: La Interculturalidad influye significativamente en el desarrollo de la Identidad Nacional en estudiantes del Colegio Emblemático n ${ }^{\circ} 56001$ "Mateo Pumacahua", Sicuani, Cusco-Perú.

Ho: La Interculturalidad NO influye significativamente en el desarrollo de la Identidad Nacional en estudiantes del Colegio Emblemático no 56001 "Mateo Pumacahua", Sicuani, Cusco-Perú. (Tabla 2).

Tabla 2. Nivel de significancia (nivel de riesgo) $\alpha=0,05$ (5\%).

\begin{tabular}{lllcc}
\hline & & Interculturalidad & Identidad Nacional \\
\hline $\begin{array}{l}\text { Rho de } \\
\text { Spearman }\end{array}$ & Interculturalidad & Coeficiente de correlación & 1,000 &, $901^{* *}$ \\
& & Sig. (bilateral) & $\cdot$ &, 000 \\
& $\mathrm{~N}$ & 217 & 217 \\
& & Coeficiente de correlación &, $901^{* *}$ & 1,000 \\
& Identidad Nacional & Sig. (bilateral) &, 000 & $\cdot$ \\
& & $\mathrm{N}$ & 217 & 217 \\
\hline
\end{tabular}

** La correlación es significativa en el nivel 0,01 (bilateral).

Coeficiente de correlación: 0,901 Sig. (bilateral / p valor) 0,000

Regla de decisión: Deberemos aceptar Ho si: Sig. (p valor) $\geq \alpha$, y rechazar Ho si: Sig. ( $p$ valor) $<\alpha$.

Decisión Estadística: Puesto que: 0,000 $<0,05$ se acepta la hipótesis del investigador $(\mathrm{Hi})$ y se rechaza la hipótesis nula (Ho).

\section{Contrastación de las hipótesis específicas:}

Hipótesis específica 1: Las prácticas culturales influyen significativamente en el desarrollo de la Identidad Nacional en estudiantes del Colegio Emblemático no 56001 "Mateo Pumacahua", Sicuani, Cusco-Perú.

Se plantean las siguientes hipótesis de trabajo: 
Hi: Las prácticas culturales influyen significativamente en el desarrollo de la Identidad Nacional en estudiantes del Colegio Emblemático no 56001 "Mateo Pumacahua", Sicuani, Cusco-Perú.
Ho: Las prácticas culturales influyen $\mathrm{NO}$ influyen significativamente en el desarrollo de la Identidad Nacional en estudiantes del Colegio Emblemático no 56001 "Mateo Pumacahua", Sicuani, Cusco-Perú. (Tabla 3).

Tabla 3. Nivel de significancia (nivel de riesgo) $\alpha=0,05$ (5\%).

\begin{tabular}{lllcc}
\hline & & Prácticas Culturales & Identidad Nacional \\
\hline Rho de & Prácticas culturales & Coeficiente de correlación & 1,000 &, $801^{* *}$ \\
& Sig. (bilateral) & $\cdot$ &, 031 \\
& $\mathrm{~N}$ & 217 & 217 \\
& & Coeficiente de correlación &, $801^{\star *}$ & 1,000 \\
& Identidad Nacional & Sig. (bilateral) &, 031 & $\cdot$ \\
& $\mathrm{N}$ & 217 & 217 \\
\hline
\end{tabular}

**. La correlación es significativa en el nivel 0,01 (bilateral).

Coeficiente de correlación: 0,801 Sig. (bilateral / p valor) 0,031

Regla de decisión: Deberemos aceptar Ho si: Sig. (p valor) $\geq \alpha$, y rechazar Ho si: Sig. (p valor) $<\alpha$.

Decisión Estadística: Puesto que: 0,031 < 0,050 se acepta la hipótesis del investigador $(\mathrm{Hi})$ y se rechaza la hipótesis nula (Ho).

Hipótesis específica 2: El uso del idioma quechua influye significativamente en el desarrollo de la Identidad Nacional en estudiantes del Colegio Emblemático $\mathrm{n}^{\circ} 56001$ "Mateo Pumacahua", Sicuani, Cusco-Perú.
Se plantean las siguientes hipótesis de trabajo:

Hi: El uso del idioma quechua influyen significativamente en el desarrollo de la Identidad Nacional de los estudiantes del Colegio Emblemático no 56001 "Mateo Pumacahua", Sicuani - Cusco.

Ho: El uso del idioma quechua $\mathrm{NO}$ influyen significativamente en el desarrollo de la Identidad Nacional de los estudiantes del Colegio Emblemático no 56001 "Mateo Pumacahua", Sicuani - Cusco. Las variables en cuestión son independientes. (Tabla 4). 
Tabla 4. Nivel de significancia (nivel de riesgo) $\alpha=0,05$ (5\%).

\begin{tabular}{lllcc}
\hline & & $\begin{array}{c}\text { Uso del idioma } \\
\text { quechua }\end{array}$ & Identidad Nacional \\
\hline $\begin{array}{l}\text { Rho de } \\
\text { Spearman }\end{array}$ & Uso del idioma quechua & Coeficiente de correlación & 1,000 &, $770^{* *}$ \\
& & Sig. (bilateral) & $\cdot$ &, 002 \\
& $\mathrm{~N}$ & 217 & 217 \\
& & Coeficiente de correlación &, $770^{* *}$ & 1,000 \\
& Identidad Nacional & Sig. (bilateral) &, 002 &. \\
& $\mathrm{~N}$ & 217 & 217 \\
\hline
\end{tabular}

**. La correlación es significativa en el nivel 0,01 (bilateral).

Coeficiente de correlación: 0,770 Sig. (bilateral / p valor) 0,002

Regla de decisión: Deberemos aceptar Ho si: Sig. (p valor) $\geq a$, y rechazar Ho si: Sig. ( $p$ valor) $<\alpha$.

Decisión Estadística: Puesto que: 0,002 <0,050 se acepta la hipótesis del investigador $(\mathrm{Hi})$ y se rechaza la hipótesis nula (Ho).

Hipótesis específica 3: Los procesos de convivencia, cooperación y autoestima influyen significativamente en el desarrollo de la Identidad Nacional en estudiantes del Colegio Emblemático no 56001 "Mateo Pumacahua", Sicuani - Cusco.
Se plantean las siguientes hipótesis de trabajo:

Hi: Los procesos de convivencia, cooperación y autoestima, influyen significativamente en el desarrollo de la Identidad Nacional de los estudiantes del Colegio Emblemático n 56001 "Mateo Pumacahua", Sicuani - Cusco.

Ho: Los procesos de convivencia, cooperación y autoestima, NO influyen significativamente en el desarrollo de la Identidad Nacional de los estudiantes del Colegio Emblemático ${ }^{\circ}$ 56001 "Mateo Pumacahua”, Sicuani - Cusco. (Tabla 5).

Tabla 5. Nivel de significancia (nivel de riesgo) $\alpha=0,05$ (5\%).

\begin{tabular}{|c|c|c|c|c|}
\hline & & & $\begin{array}{c}\text { Procesos de convivencia, } \\
\text { cooperación y } \\
\text { autoestima }\end{array}$ & Identidad Nacional \\
\hline \multirow[t]{6}{*}{$\begin{array}{l}\text { Rho de } \\
\text { Spearman }\end{array}$} & $\begin{array}{l}\text { Procesos de convivencia, } \\
\text { cooperación y autoestima }\end{array}$ & Coeficiente de correlación & 1,000 &, $831^{\star *}$ \\
\hline & & Sig. (bilateral) & . &, 004 \\
\hline & & $\mathrm{N}$ & 217 & 217 \\
\hline & Identidad Nacional & Coeficiente de correlación &, $831^{\star \star}$ & 1,000 \\
\hline & & Sig. (bilateral) & ,004 & . \\
\hline & & $\mathrm{N}$ & 217 & 217 \\
\hline
\end{tabular}

\footnotetext{
**. La correlación es significativa en el nivel 0,01 (bilateral).
} 
Coeficiente de correlación: 0,831 Sig. (bilateral / p valor) 0,004

Regla de decisión: Deberemos aceptar Ho si: Sig. (p valor) $\geq \alpha$, y rechazar Ho si: Sig. (p valor) $<\alpha$.

Decisión Estadística: Puesto que: 0,004 < 0,050 se acepta la hipótesis del investigador $(\mathrm{Hi})$ y se rechaza la hipótesis nula (Ho).

\section{Discusión}

En base a estos resultados y a los estadísticos presentadosenlapruebadehipótesis (sig.0,000<0,05) concluimos que: las prácticas de Interculturalidad (Prácticas culturales; Uso del idioma quechua; Procesos de convivencia, cooperación y autoestima) sí, influyen significativamente en el desarrollo de la Identidad Nacional de los estudiantes del Colegio Emblemático no 56001 "Mateo Pumacahua", Sicuani, Cusco en Perú.

Estos resultados se asemejan con la investigación realizada por Panduro Coral (2017) quien escribió su tesis Programa de interiorización para conservar la identidad cultural en los estudiantes de Educación Primaria de Lamas - San Martín, quien concluyó: que los estudiantes interiorizan su cultura y se identifican a través de actividades como: Festivales, eventos culturales programas artísticos, Foros educativos, ferias gastronómicas, exposiciones artesanales. Se evidencia que las creencias, costumbres, modos de vida tradiciones, comidas, artesanía, actos ceremoniales son rescatadas y valoradas por los estudiantes.

Lo anteriormente planteado se alinea a la teoría propuesta por De la Hidalga (2019), quien manifiesta que: la interculturalidad es una propuesta educativa alternativa interdisciplinaria, participativa e interactoral en una región con población indígena y mestiza que se ubica en el marco del cuestionamiento de una educación escolar con perspectiva monocultural y discriminatoria que invisibiliza la diversidad cultural.

\section{CONCLUSIONES}

Luego de observar los resultados del instrumento "Identidad Nacional" se determinó que son logradas de forma "buena" por 116 de los estudiantes, de manera "regular" por 40 estudiantes y de forma "mala" por 61 estudiantes. Por tanto, se puede concluir que la "Identidad nacional" es lograda de manera "buena" por la mayoría de los estudiantes. En base a estos resultados y a los estadísticos presentados en la prueba de hipótesis (sig. 0,000<0,05) se finaliza que el desarrollo de la Identidad Nacional (Identificación con los símbolos patrios; Identificación con los monumentos históricos; identificación con los personajes), son el efecto positivo de que la Interculturalidad utilizada por los profesores, influyen de forma significativa en el desarrollo de la Identidad Nacional de los estudiantes del Colegio Emblemático no 56001 Mateo Pumacahua.

\section{REFERENCIAS}

Aguado, T. (2005). La educación intercultural en la práctica escolar. Investigación en el ámbito español. Revista de Educación. https://core. ac.uk/download/pdf/41562895.pdf

Beramendi, M., Espinosa, A., y Acosta, Y. (2020). Percepción del Sistema Normativo y sus Correlatos Psicosociales en Argentina, Perú y Venezuela. Revista Colombiana de Psicología, 29, 13-27. https://doi.org/10.15446/rcp. v29n1.75797

Bueno, G; Molina Luz M y Porras, A. (2009). Estrategias pedagógicas orientadas a fortalecer el respeto hacia los símbolos patrios en los estudiantes de quinto grado sección "b" en la Escuela Bolivariana Barrancas. http://www. daleya.com/?query=tesis+sobre+identidad+ 
c o n $+\mathrm{los}+\mathrm{simbolos}+\mathrm{p}$ a t r i o s \& d a l e y a idext $\% 5$ B \% 5 D = $1 \&$ daleyaidext $\% 5 \mathrm{~B} \% 5 \mathrm{D}=2 \& \mathrm{xt}=$

Cueto, R., Espinosa, A. y Robles, R. (2017). Narrativas sobre la sociedad peruana y la identidad nacional en universitarios peruanos. Límite. Revista Interdisciplinaria de Filosofía y Psicología, 12(38), 07-21

De la Hidalga Ledesma, V. (2019). Interculturalidad en la Universidad Veracruzana. Aprendizaje entre diversidad de saberes académicos y comunitarios hacia el diálogo (Doctoral dissertation, Universidad Veracruzana. instituto de Investigación en Educación. Región Xalapa)

Diario El Peruano. (04 de enero de 2017). Diario El Peruano. Obtenido de https://busquedas. elperuano.pe/normaslegales/actualizacion-delcapital-socialminimo-de-las-empresas-supecircular-no-g-190-2017-1470996-1/

Espinosa, A., Acosta, Y., Valencia, J., Vera, Á., Soares da Silva, A., Romero, J. C. yBeramendi, M. (2016). Calidez, competencia, moralidad y nacionalismo ideal como dimensiones autoestereotípicas en seis países de Latinoamérica. Avances en Psicología Latinoamericana, 34(2), 395-413

INDEPA (2010). Aportes para un enfoque intercultural. Lima: Instituto Nacional de Desarrollo de Pueblos Andinos, Amazónicos y Afroperuano

Fernández, M. Z. (2008). Percepción de la interculturalidad en un programa de educación comunitaria. [Tesis Licenciatura, PUCP]. http://tesis.pucp.edu.pe/repositorio/ handle/123456789/693
Panduro Coral, M. E. (2017). Programa de interiorización para conservar la identidad cultural en los estudiantes de Educación Primaria de Lamas-San Martín

Pastor, G. (2006). Estudio sobre la afirmación de la identidad nacional en el Perú. Revista temática centrodealtos estudiosnacionales.https://www2. congreso.gob.pe/ sicr/cendocbib/con5_uibd. nsf/ 724B14F44EA54588052582BE00770AD7 /\%24FILE/ART4-G-Pastor.pdf

Pérez-Rodríguez, I. L. (2012). Identidad nacional y sentidos de los jóvenes sobre su nación. Revista Latinoamericana de Ciencias Sociales, Niñez y Juventud, 10 (2), pp. 871-882. http://www.scielo. org.co/pdf/rlcs/v10n2/v10n2a07.pdf

Romero, E. (2015). ¿Existe nación en el Perú? Hispanic American Historical Review. http:// hahr-online.com/existe-nacion-en-el-peru/

Salazar, M., y Schmitz, V. (2015). Prácticas educativas en el nivel de inicial desde la educación intercultural. [Tesis Licenciatura, PUCP]. https://tesis.pucp.edu.pe/repositorio/ handle/20.500.12404/6468

Walsh, C. (2009). Interculturalidad, Estado, Sociedad: Luchas (de) coloniales de nuestra época. Quito: Universidad Andina Simón Bolívar 